University of Nebraska - Lincoln

DigitalCommons@University of Nebraska - Lincoln

\title{
A Comparative Study of Geometric Rule Learning by Nutcrackers (Nucifraga columbiana), Pigeons (Columba livia), and Jackdaws (Corvus monedula)
}

Juli E. Jones

University of Nebraska - Lincoln

Elena Antoniadis

University of Toronto

Sara J. Shettleworth

University of Toronto

Alan Kamil

University of Nebraska - Lincoln, akamil1@unl.edu

Follow this and additional works at: https://digitalcommons.unl.edu/bioscibehavior

Part of the Behavior and Ethology Commons

Jones, Juli E.; Antoniadis, Elena; Shettleworth, Sara J.; and Kamil, Alan, "A Comparative Study of Geometric Rule Learning by Nutcrackers (Nucifraga columbiana), Pigeons (Columba livia), and Jackdaws (Corvus monedula )" (2002). Papers in Behavior and Biological Sciences. 70.

https://digitalcommons.unl.edu/bioscibehavior/70

This Article is brought to you for free and open access by the Papers in the Biological Sciences at DigitalCommons@University of Nebraska - Lincoln. It has been accepted for inclusion in Papers in Behavior and Biological Sciences by an authorized administrator of DigitalCommons@University of Nebraska - Lincoln. 
Published in Journal of Comparative Psychology 116:4 (2002), pp. 350-356; doi: 10.1037/0735-7036.116.4 Copyright ( 2002 American Psychological Association. Used by permission. "This article may not exactly replicate the final version published in the APA journal. It is not the copy of record." http://www.apa.org/journals/jcp/

This research was supported by grants from the National Science Foundation and the Natural Sciences and Engineering Research Council. We thank David Kabelik for help with Experiment 2, Andy Gristock for pigeon care, and Alex Kacelnik for assistance in obtaining the jackdaws.

Submitted June 11, 2001; revised February 6, 2002; accepted February 9, 2002.

\title{
A Comparative Study of Geometric Rule Learning by Nutcrackers (Nucifraga columbiana), Pigeons (Columba livia), and Jackdaws (Corvus monedula)
}

\author{
Juli E. Jones, School of Biological Sciences, University of Nebraska-Lincoln \\ Elena Antoniadis, Department of Psychology, University of Toronto \\ Sara J. Shettleworth, Department of Psychology, University of Toronto \\ Alan C. Kamil, School of Biological Sciences, University of Nebraska-Lincoln \\ Corresponding author - Alan C. Kamil, School of Biological Sciences, University of Nebraska-Lincoln, \\ Lincoln, Nebraska 68588-0118; email akamil@unlserve.unl.edu. \\ Juli E. Jones is now at the Neuroscience and Behavior Program, University of Massachusetts at Amherst.
}

\begin{abstract}
Three avian species, a seed-caching corvid (Clark's nutcrackers; Nucifraga columbiana), a non-seed-caching corvid (jackdaws; Corvus monedula), and a non-seed-caching columbid (pigeons; Columba livia), were tested for ability to learn to find a goal halfway between 2 landmarks when distance between the landmarks varied during training. All 3 species learned, but jackdaws took much longer than either pigeons or nutcrackers. The nutcrackers searched more accurately than either pigeons or jackdaws. Both nutcrackers and pigeons showed good transfer to novel landmark arrays in which interlandmark distances were novel, but inconclusive results were obtained from jackdaws. Species differences in this spatial task appear quantitative rather than qualitative and are associated with differences in natural history rather than phylogeny.
\end{abstract}

A central question in the study of animal behavior is how landmarks are used to orient and navigate in space (Cheng \& Spetch, 1998; Collett \& Zeil, 1996). Honeybees relocate a goal such as a source of nectar by moving so as to match the currently perceived visual image with the remembered image of the landmark array (Cartwright \& Collett, 1983; Collett \& Zeil, 1998). Under some circumstances, rats use the overall shape of a space as defined by objects and surfaces (Cheng, 1986; Margules \& Gallistel, 1988). Pigeons and other birds often remember distances and/or direction between landmarks and goal locations (Cheng, 1995; Gould-Beierle \& Kamil, 1996; Kamil \& Cheng, 2001). All of these examples involve responding to specific distances and/or directions among objects or features in the environment (see Cheng \& Spetch, 1998). However, there are experimental results that suggest that animals can use abstract geometric relationships among landmarks or between goals and landmarks. For example, there are neurons in rat hippocampus that appear to respond to proportional distance (O'Keefe \& Burgess, 1996), and several studies have obtained behavioral evidence of search based on relative location in an environment (e.g., Cheng, 1986; Kelly, Spetch, \& Heth, 1998; Tommasi \& Vallortigara, 2000; Vallortigara, Zanforlin, \& Pasti, 1990).

The use of the geometric relationship halfway, or middle, has been investigated in several studies, with varying results. Spetch and her colleagues (Spetch, Cheng, \& MacDonald, 1996; Spetch et al., 1997) trained humans and pigeons to find a reward in the center of a square array of landmarks. Subjects were trained with a single arrangement of the landmarks for many trials until they could locate the reward accurately. Then probe tests were conducted during which the landmark array was transformed so that it formed either a rectangle or a square much larger than the square used in training. The humans still searched in the center of the new arrays, but the pigeons tended to search off center in a manner that preserved the original angular and/or distance relationships to one or two of the landmarks. This suggests that the pigeons encoded these specific relationships rather than any general rule.

In contrast, Kamil and Jones $(1997,2000)$ trained Clark's nutcrackers to search halfway between two landmarks. However, in their studies, unlike those of Spetch and her colleagues (Spetch et al., 1996, 1997), the distance between the landmarks varied during training. When presented with new interlandmark distances during probe tests, the nutcrackers generalized and continued to search halfway between the landmarks. It appears that the nutcrackers learned to search using a rule that was not dependent on the specific goal-landmark distances used during training and may have learned something akin to a general concept of "halfway."

The differences in results between experiments with nutcrackers and experiments with pigeons could be due to any of several methodological differences, including number of landmarks and the distances between them. The most likely, how- 
ever, appears to be the difference in the number of training arrays that were used. The pigeons were trained with a single array so that both absolute and relative relationships between goal and landmarks were held constant. In contrast, the nutcrackers were trained with several different arrays arranged so that absolute goal-landmark distance varied, but relative goallandmark distance was constant. Thus the pigeons could learn to find the location of the goal by learning the absolute distance and/or direction from one or two of the landmarks in the array. In contrast, no single metric based on absolute distance and/or direction would suffice for the nutcrackers. The use of several interlandmark distances during training may have forced the nutcrackers to use a search rule that fit all of them. If this was the case, then nutcrackers would generalize and pigeons would not because of differences in their training regime.

However, the difference in the results of the previous studies also could be due to a species difference. Nutcrackers and pigeons differ dramatically in their use of cached food. Clark's nutcrackers store tens of thousands of seeds in the ground each fall in thousands of different locations (Vander Wall \& Balda, 1977), whereas pigeons do not cache food. Results from many studies demonstrate that there are species differences in spatial cognition associated with species differences in caching (see review in Shettleworth, 1998), and these differences have been found in a variety of test situations, including operant tasks (Olson, Kamil, Balda, \& Nims, 1995) and an analog of the radial maze (Kamil, Balda, \& Olson, 1994; Gould-Beierle, 2000). Nutcrackers may also differ in the extent to which they abstract a general rule from experience with a restricted set of stimuli (Mackintosh, 1988). Corvids other than nutcrackers (rooks and crows) have transferred to new stimuli in a delayed matching-to-sample task much better than pigeons (Wilson, Mackintosh, \& Boakes, 1985), although pigeons do show greater transfer when trained with a number of different sample stimuli than with a single pair (e.g., Wright, Cook, Rivera, Sands, \& Delius, 1988).

There may also be a specific connection between natural history and performance in hidden-goal tasks. The survival and reproduction of nutcrackers depend on their ability to relocate their hidden seeds. Many of their caches are distributed across alpine meadows, far from landmarks. As a result, nutcrackers regularly face the problem of locating a goal on the basis of relatively distant landmarks. In contrast, pigeons do not face this problem. Thus tasks that require finding a specific location on the basis of a landmark array may be more appropriate for Clark's nutcrackers than for pigeons.

In light of these differences in natural history and in performance in earlier studies of spatial cognition, we compared the ability of pigeons and nutcrackers to find the center of novel arrays of landmarks after very similar training. During Experiments 1 and 2, Clark's nutcrackers and pigeons were trained to find food hidden halfway between two landmarks separated by several distances, as in the studies by Kamil and Jones (1997, 2000). The birds were then tested with a variety of novel interlandmark distances. These transfer tests included tests with interlandmark distances beyond those used during training. Searching in the middle of the array during such tests cannot be readily explained in terms of use of specific vectors from individual landmarks (Biegler, McGregor \& Healy, 1998).

Although the differences in natural history between nutcrackers and pigeons lead to the prediction that nutcrackers will perform better than pigeons, it would be difficult to attribute such a species difference to the difference in dependence on stored food. Pigeons and nutcrackers are very distantly related and differ in many ways. Therefore, an additional comparison is needed, either with a close relative of the pigeon that depends on cached food or with a close relative of the nutcracker that does not. We know of no columbid that caches, but there are several corvids that cache little, if at all. One of these is the jackdaw, a well-studied corvid rarely observed to cache in the field. Jackdaws are closely related to Clark's nutcrackers within the Corvidae (Hope, 1989), and they perform worse than nutcrackers, pinyon jays, and scrub jays in a radial arm maze analog test of spatial memory (Gould-Beierle, 2000).

This combination of close relatedness to nutcrackers, lack of natural caching behavior, and poor performance in a spatial memory task made jackdaws a logical choice for testing in Experiment 3. If the performance of nutcrackers reflects a form of spatial cognition shared by all corvids, jackdaws should perform like nutcrackers, but if birds that store food have a distinctive ability to use geometric relationships among landmarks, the jackdaws would be expected to perform like pigeons. Finally, if training with a variety of interlandmark distances facilitates transfer to the new interlandmark distances, birds of all three species would be expected to show a similar pattern of results.

While Clark's nutcrackers and jackdaws were tested at the University of Nebraska-Lincoln, pigeons were tested at the University of Toronto. This was partially because testing pigeons and corvids in the same apparatus or room carries some health risks for the birds. Each group may well carry diseases relatively harmless to itself but dangerous to others (Clubb, 1997). In addition, the different labs are each experienced with corvid and columbid species, respectively, and best equipped to test them.

\section{Experiment 1-Clark's Nutcrackers}

\section{Method}

Subjects - Five experimentally naive Clark's nutcrackers (Nucifraga columbiana) served as subjects. The birds were individually housed in a colony room maintained at $22{ }^{\circ} \mathrm{C}$ on a 14:10-hr light-dark cycle. Throughout the course of the experiment the birds were kept at approximately $90 \%$ of their free-feeding weight by controlled daily feedings of turkey starter, parrot pellets, mealworms, sunflower seeds, pine seeds, and vitamin supplements. They had unlimited access to water and grit in their home cages.

Apparatus - The experiment was conducted in a 4.4-m (north-south; NS) $\times$ 2.7-m (east-west; EW) observation room illuminated by four centrally located fluorescent lights. Birds were placed in a holding cage, located behind a porthole, before and between trials. The birds entered the room through the porthole on the east wall just below a smoked-glass observation window with a drawn curtain. The observer entrance door was north of the porthole on the east wall. A Panasonic video camera (model WV-BL200) was mounted above the suspended ceiling near the center of the room and connected to a video monitor and VCR (model JVC BR-3200U). Subjects were monitored through the observation window and on the video screen. A $7-\mathrm{cm}$ high wooden floor was built on top of the room's concrete floor. This raised floor began $90 \mathrm{~cm}$ from the east wall and extended the width of the room to the west wall and was covered with a 2-cm layer of cellulose substrate.

There were two 40 -cm-high landmarks $(3.4 \mathrm{~cm}$ diameter blue and red PVC pipe) that were always placed north (red) and south (blue) of each other. The distance between the landmarks varied in increments of $20 \mathrm{~cm}$ from 40 to $100 \mathrm{~cm}$ for training and from 30 to $110 \mathrm{~cm}$ for subsequent transfer tests. The position of the landmark array varied from trial to trial. The array was always placed so that the goal location was 
within the rectangle that extended $60 \mathrm{~cm}$ east and west and $40 \mathrm{~cm}$ north and south of the center of the experimental arena. This allowed for 116 different goal locations. The room was divided into four equal quadrants, and the landmark array was placed in each quadrant once per day for each bird, in random order. Once every room position had been used, the positions and quadrants were re-randomized.

Familiarization - The birds were made familiar with the experimental room for 5 days with two trials per day. The first 2 days, the birds entered the room and retrieved two unshelled pine seeds. The seeds were placed on a dish consisting of a 3.5-cm diameter plastic lid from a $35-\mathrm{mm}$ film container. For the first 2 days the dish and seeds (completely exposed) were placed on top of the substrate. The next 3 days, the dish was filled with substrate that completely covered the seeds to train the birds to use the dish as a cue to seed location. For each trial, following the first retrieval, the dish was moved to random locations within the room.

Training procedure - The birds were brought individually from their home cage to the holding cage outside the observation room. Initially, the lights in the holding room were off and the lights in the observation room were on. The sliding door in the porthole was opened, and a bird was allowed to enter the room. At the end of the trial, the observation room lights were turned off, the sliding door was opened, the holding room lights were turned on, and the bird flew back to the holding cage. Trials continued until the goal was located, 40 probes were made, or the bird had been in the room for $10 \mathrm{~min}$. The definition of a probe was the point at which a bird's beak came into contact with the substrate. On the instances in which a bird did not find the seed within 40 probes, the trial was terminated and the bird was not allowed another attempt at that position. After the completion of four trials, the bird was taken back to its home cage.

The experiment was conducted in two stages, training (acquisition) and transfer testing. Four different interlandmark distances were used during training: 40, 60, 80, and $100 \mathrm{~cm}$. Each interlandmark distance was presented in random order once a day, and there were two types of trials. During exposed trials, a part of the dish on which the seed was placed was exposed and easily visible. During buried-seed trials, the seed and dish were completely buried. During the first part of the acquisition stage, each bird experienced three exposed trials each day preceding one buried-seed trial. The buried-seed trials were organized into a completely randomized block design, and the interlandmark distances not presented in the buried-seed trial each day were randomly assigned to the exposed trials. Each session was videotaped, and behavior during buried-seed trials was analyzed. A total error score was calculated for each bird each session (as described below) to provide acquisition data. For each nutcracker, Stage 1 continued for a minimum of 30 buried-seed trials and until the bird's search behavior met the criterion of a 10-day period during which the mean total error score was less than $25 \mathrm{~cm}$ and at least $60 \%$ of the seeds were located during buried seed trials.

During the second phase of acquisition, the first 2 trials each day were exposed trials, and Trials 3 and 4 were buried-seed trials. For each bird, this stage continued until a 10-trial block of buried-seed trials occurred during which total error score was less than $20 \mathrm{~cm}$ and the seed was found on at least 8 of 10 buried-seed trials. During the final phase of acquisition, all 4 trials were buried-seed trials. When a bird's performance met the criterion of no difference in search error among the last four 10-day blocks of buried-seed trials, indicating asymptotic performance had been reached, acquisition was complete and transfer testing began.

During the next phase, no-seed trials were introduced to accustom the birds to occasional nonreward. During no-seed trials, neither the dish nor the seeds were placed in the room, and the trial ended after the bird made five probes. The no-seed trial was randomly assigned to the second, third, or fourth trial each day. This phase lasted for 8 days during which each bird received two no-seed trials at each training interlandmark distance.

The next stage was transfer testing, which lasted 27 days. Each day each bird received three buried-seed trials and one no-seed trial. The no-seed trial was randomly assigned to Trials 2,3 , or 4 each day. Training interlandmark distances were used for all buried-seed trials. Noseed trials were conducted with the five new $(30,50,70,90$, and 110 $\mathrm{cm})$ and the four old $(40,60,80$, and $100 \mathrm{~cm})$ interlandmark distances, in a randomized block design. Each interlandmark distance was presented before another interlandmark distance could be repeated, and all no-seed trials were analyzed.

Determining position of digs - To determine the locations at which birds searched, we analyzed each session by reviewing the videotaped trial on a Panasonic VCR (model AG-1730) that allowed slowmotion, freeze-frame playback attached to a TARGA videograph system (Truevision Inc., Indianapolis, IN). A digitizing pad was used to record the locations of the landmarks, goal, and dig locations. The first 10 digs were recorded on buried-seed trials (unless the seed was found in less than 10 digs), and all 5 digs were recorded on no-seed probe trials. The TARGA videograph system assigned an EW and an NS coordinate for each marked location. For analysis, the coordinates for each of the first 5 digs were subtracted from those of the goal location and converted to centimeters. The absolute coordinate values were then averaged. EW error was in the axis perpendicular to the line that connected the landmarks, whereas NS error was in the axis error parallel to the line that connected the landmarks. The EW and NS error for each dig was used to calculate search error using the Pythagorean theorem. Data analysis was carried out by analyses of variance (ANOVAs; with $\alpha=.05$ ). If overall $F$ ratios were significant, Fisher's least significant differences (LSDs) were used for subsequent tests.

\section{Results}

All birds completed acquisition in 100 or 110 buried-seed trials. These trials were divided into blocks of 10 trials each and were analyzed with a Subject $\times$ Block repeated measures ANOVA on search error during the first 10 blocks. There was a significant decrease in search error across blocks, $F(9,36)$ $=2.91, p<.01$ (see Figure 1). At asymptote (during the last 3 blocks of acquisition), mean search error ( \pm SE) was $11.98 \pm$ $2.13 \mathrm{~cm}$ (see Table 1).

Performance during the introduction of no-seed trials was analyzed with a Bird $\times$ Condition ANOVA comparing search error on buried-seed trials with that on no-seed trials. Mean search error $( \pm S E)$ was $8.68 \pm 0.87 \mathrm{~cm}$ when a seed was present and $10.28 \pm 2.08 \mathrm{~cm}$ when no seed was present, $F(1,4)<$ 1. Therefore we pooled data across all 32 trials of this stage and used these data to examine the effects of interlandmark distance on performance before transfer-testing began. The search error data were analyzed with an Interlandmark Distance $\times$ Bird ANOVA. Search error tended to increase as the distance between the landmarks increased, but the effect only approached significance, $F(3,12)=3.34, p<.06$.

Search error during transfer testing (see Figure 1 ) was analyzed with a Bird $\times$ Interlandmark Distance $\times$ Axis (EW vs. NS) ANOVA. As distance between the landmarks increased, error distance also increased, $F(8,32)=7.31, p<.01$. There were no significant effects of axis, $F(1,4)=4.06, p>.10$, and the Axis $\times$ Interlandmark Distance interaction was also not significant, $F(8$, $32)=1.37, p>$.20. A Fisher's LSD test was used to compare performance at each interlandmark distance against performance at the next higher or lower interlandmark distance. None of the eight individual comparisons was significant. In addition, we also divided the interlandmark distances into three conditions: those used during training, those outside of the training range, and the novel distances within the training range. These data were analyzed with a Condition $\times$ Bird repeated measures ANOVA, and the result was not significant, $F(2,8)<1$. 

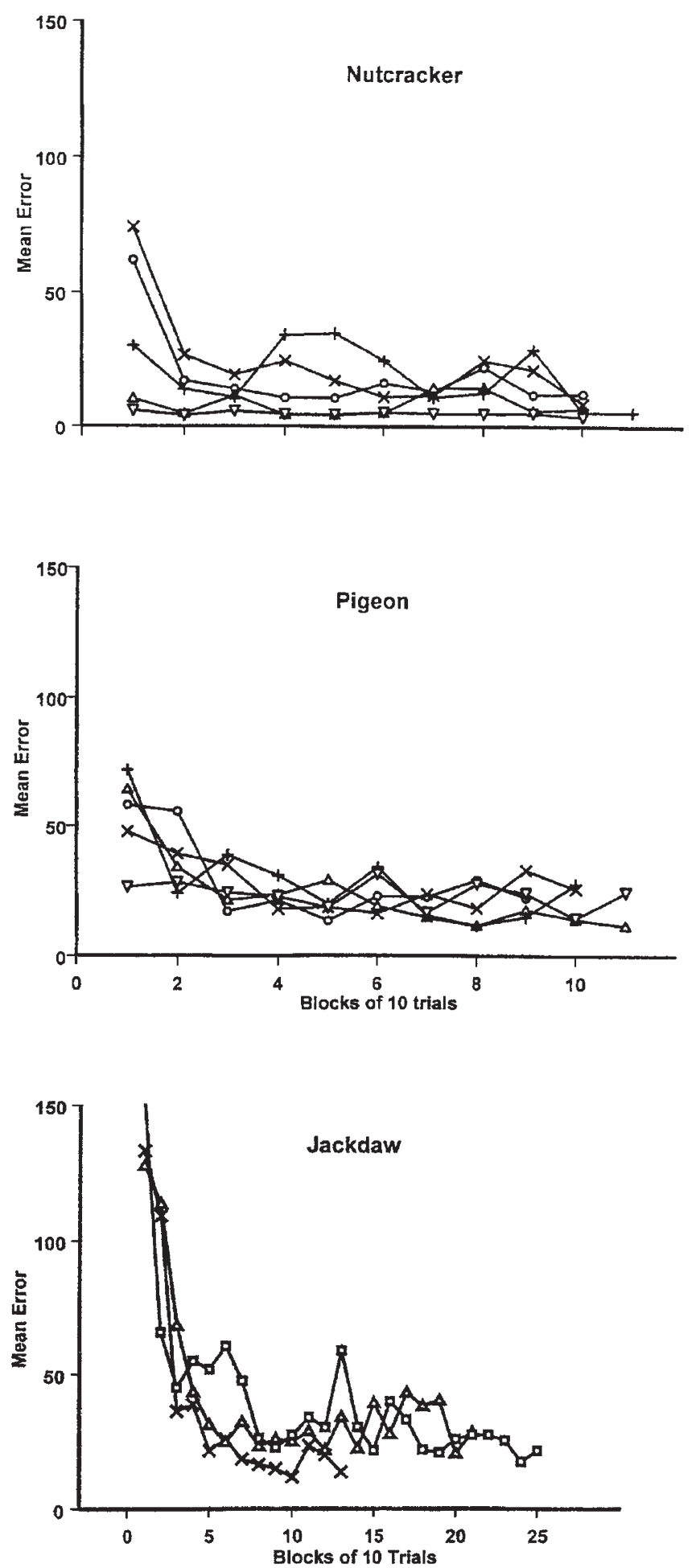

Figure 1. Mean precision of search during acquisition for each bird (indicated by different symbols) in Experiments 1-3 during each block of 10 trials. Top: nutcrackers; middle: pigeons; bottom: jackdaws. Note that the $\mathrm{x}$-axis scale for jackdaws differs from others.

\section{Discussion}

These results were very similar to those reported by Kamil and Jones (1997, 2000). The nutcrackers readily learned to find a seed buried halfway between two landmarks. Dur-
Table 1. Performance of Each Species, With 95\% Confidence Intervals, During the Last Four Blocks of Acquisition During Experiments 1-3

\begin{tabular}{lcccc}
\hline Species & $\begin{array}{c}\text { Mean error } \\
\text { asymptote } \\
(\mathrm{cm})\end{array}$ & $\begin{array}{c}95 \% \\
\text { confidence } \\
\text { interval }\end{array}$ & $\begin{array}{c}\text { Mean no. } \\
\text { of trials to } \\
\text { criterion }\end{array}$ & $\begin{array}{c}95 \% \\
\text { confidence } \\
\text { interval }\end{array}$ \\
\hline Nutcracker & 11.98 & $7.41-16.55$ & 102.00 & $96.45-107.55$ \\
Pigeon & 21.10 & $17.41-24.79$ & 102.00 & $91.61-112.39$ \\
Jackdaw & 23.44 & $17.53-29.35$ & 196.67 & $44.88-348.45$ \\
\hline
\end{tabular}

ing acquisition, their search error decreased from approximately $35 \mathrm{~cm}$ at the beginning of training to $12 \mathrm{~cm}$ at the end of training and to about $8 \mathrm{~cm}$ by the end of transfer testing. The nutcrackers also generalized readily to new interlandmark distances both inside and outside the range of interlandmark distances used during training. Performance at training interlandmark distances was never significantly better than performance at any next higher or lower transfer distance. This offers strong support for the hypothesis that the birds had learned a single general principle rather than memorized the four training landmark-goal distances or vectors (Biegler et al., 1998).

\section{Experiment 2-Pigeons}

\begin{abstract}
Method
Subjects - Seven white king pigeons (Columba livia) 2.5-3.5 years of age began the experiment. The pigeons had previously served in a discrimination-learning task in an open field that did not involve the use of landmarks for food retrieval. Two pigeons were dropped because of difficulty during training. The animals were housed individually in wire mesh cages $(36 \times 35 \times 41 \mathrm{~cm})$, kept on a 14:10-hr light-dark cycle, and maintained at $22{ }^{\circ} \mathrm{C}$. The birds were maintained at $85 \% \pm$ $2 \%$ of their free-feeding weight through daily controlled feedings of mixed grain. Subjects were allowed unlimited access to water and grit in their home cages.
\end{abstract}

Apparatus - The experimental room and apparatus closely resembled those in Experiment 1 with the following exceptions. First, the experimental room was $3.5 \times 3.0 \mathrm{~m}$. The observation window was west of the entrance point and both were located on the $3.5 \mathrm{~m}$ south wall. All trials were videotaped by a Panasonic camera (model TX62045) centered in the ceiling of the room and connected to a VCR (model JVC HR-VP648U). Two $40 \mathrm{~cm}$ high, $2.5 \mathrm{~cm}$ diameter wooden cylinders painted red (north) and blue (south) served as landmarks. They stood on flat pieces of wood buried beneath the bedding. A $30-\mathrm{cm} \times$ $21-\mathrm{cm} \times 77-\mathrm{cm}$ stand positioned under the porthole on the south wall served as the landing platform for the bird's reentrance to the holding cage. The floor of the aviary was covered with wood chips (Beta Chip brand; Northeastern Products, Inc., Warrensburg, NY), approximately $5 \mathrm{~cm}$ deep.

Procedure - The procedure was the same as that of Experiment 1 except that familiarization required 6 days. During the first 2 days, birds entered the room two times per day and retrieved mixed grain from the food dish located halfway between the landmarks, on the surface of the bedding. For the following 4 days, increasing amounts of bedding were added to the dish until the birds were recovering seeds that were completely hidden, with only the rim of the dish showing. Approximately $8.3 \mathrm{~g}$ of seeds were given per trial in all stages. Once familiarization was complete, Experiment 2 followed the protocol used in Experiment 1. Note that this protocol specified behavioral criteria for the end of each stage, so that the number of trials per stage could vary between species. 


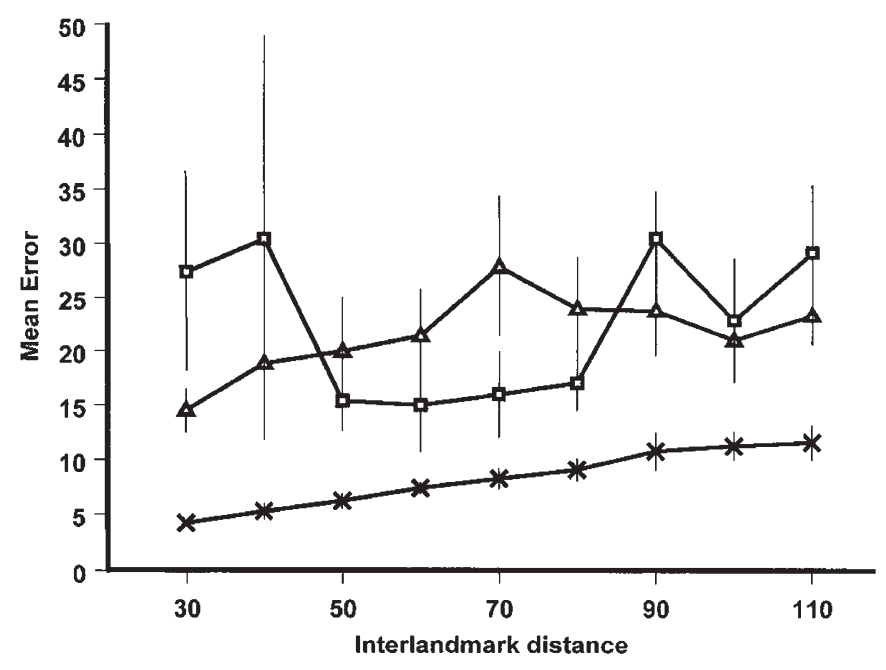

Figure 2. Mean precision of search obtained at each interlandmark distance tested during transfer testing of Experiments 1-3. $\times=$ nutcrackers; $\Delta=$ pigeons; $\square=$ jackdaws. Error bars represent $\pm 1 \mathrm{SE}$.

Determining position of probes - To determine the position of the probes, we analyzed each session by frame-by-frame playback of videotaped trials. A grid that served to measure the position of the probes was placed on the monitor screen. Each $2.5-\mathrm{cm} \times 2.5-\mathrm{cm}$ square on the grid represented a $10-\mathrm{cm} \times 10-\mathrm{cm}$ square in the real space of the experimental floor. Using this grid, the goal and probe locations were recorded for each buried-seed or no-seed trial. The first 5 digs were recorded on buried-seed trials, and all 5 digs were recorded on no-seed trials. The mathematical calculations used to determine error distance were the same as in Experiment 1 as were methods of statistical analysis.

\section{Results}

All pigeons met criterion for the completion of Stage 3 in 100-110 buried-seed trials. Search error decreased throughout Stages 1-3 of acquisition (see Figure 1). A Subject $\times$ Block repeated measures ANOVA on search error revealed a significant decrease in total error distance, $F(8,32)=3.88, p<.01$. At asymptote (during the last 3 blocks of acquisition), mean search error $( \pm S E$ ) was $21.10 \pm 1.72 \mathrm{~cm}$ (see Table 1). Performance on buried-seed trials was compared with performance on no-seed trials during the stage in which no-seed trials were introduced. Mean search error $( \pm \mathrm{SE})$ was $23.39 \pm 2.11 \mathrm{~cm}$ when seeds were present and $20.02 \pm 2.10 \mathrm{~cm}$ when no seed was present, $F(1,4)=1.72, p>.26$. We therefore pooled data across all 32 trials of this stage and carried out a Bird $\times$ Interlandmark Distance ANOVA. Interlandmark distance had no significant effects on search error, $F(3,12)<1$.

We conducted a series of analyses to examine performance when the novel interlandmark distances were introduced during transfer testing (see Figure 2). A Bird $\times$ Interlandmark Distance $\times$ Axis ANOVA revealed no significant effects, $F(8,32)$ $=1.01, p>$.40. There were no significant effects of axis, $F(1,4)$ $=1.47, \mathrm{p}>.25$, and the Axis $\times$ Interlandmark Distance interaction was also not significant, $F(8,32)=1.53, p>0.15$. An additional analysis of search error was performed in which interlandmark distance was divided into three conditions: those used during training, those outside of the training range, and the novel distances within the training range (see Figure 3). A Condition $\times$ Bird repeated measures ANOVA revealed no significant effects, $F(2,4)<1$.

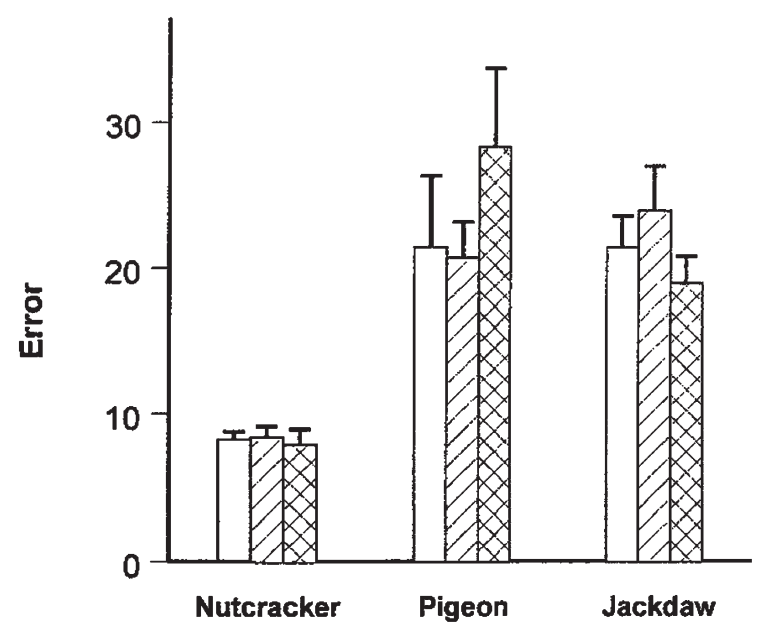

Figure 3. Mean precision of search for training (open bars), interpolated (lined bars), and extrapolated (cross-hatched bars) interlandmark distances during transfer testing of Experiments 1-3. Error bars represent \pm 1 SE.

\section{Discussion}

The results of Experiment 2 were, in many respects, parallel to those of Experiment 1. Like nutcrackers, pigeons were able to learn to search accurately when the relative relationship between a goal and landmarks was held constant but the absolute relationship varied. During transfer tests the pigeons performed as accurately with new interlandmark distances as with original training distances. Thus the pigeons demonstrated an ability to learn to search halfway between two landmarks, supporting the idea that some sort of rule had been acquired that could be generalized to novel interlandmark distances. This contrasts with the results of Spetch and her associates (Spetch et al., 1996, 1997), whose pigeons used absolute relationships during transfer tests. This supports the hypothesis that varying absolute goal-landmark distances, while holding relative distances constant produces acquisition of a principle based on the relationship between landmarks by pigeons. Further, it implies that procedures in which only a single distance is used during training leads to the reliance on absolute landmark-goal vectors. Therefore it seems that the discrepancy in transfer results between Spetch et al. $(1996,1997)$ and Kamil and Jones (1997) was due to procedural differences during training rather than species differences.

\section{Experiment 3-Jackdaws}

\section{Method}

Subjects - Five jackdaws (Corvus monedula) with previous experience in an open-room analogue of the radial maze (Gould-Beierle, 2000; Gould-Beierle \& Kamil, 1998) served as subjects for all phases of the experiment. The birds were individually housed in a colony room kept at $22{ }^{\circ} \mathrm{C}$ on a $14: 10$-hr light-dark cycle at the University of Nebraska School of Biological Sciences. Their diet consisted of turkey starter, parrot pellets, sunflower seeds, peanuts, bread, and vitamin supplements. The subjects were allowed unlimited access to water and grit in their home cages. Throughout the course of the experiment the birds were kept at approximately $90 \%$ of their free-feeding weight by controlled daily feedings. During initial training to the experimental procedures, two of the jackdaws would not dig in the substrate and were therefore dropped from the study. 
Procedure - The material, methods, and procedure were identical to that of Experiment 1. The only difference is that the reinforcement was half of a peanut. Half of a peanut and two pine nuts are approximately equivalent in weight.

\section{Results}

The jackdaws were very variable in the amount of time needed to meet the behavioral criteria, requiring 130, 210, and 250 trials to complete Stage 3. Given this variation, we did not attempt an overall analysis of search accuracy during Stages 13 , but each bird did show increased search accuracy with training (see Figure 1). During the last three blocks of acquisition, mean search error $( \pm S E$ ) was $23.44 \pm 2.56 \mathrm{~cm}$ (see Table 1$)$.

Performance on buried-seed trials was compared with performance on no-seed trials during Stage 4. Mean total search error $( \pm S E)$ was $22.89 \pm 3.19 \mathrm{~cm}$ when seeds were present and $20.80 \pm 2.74 \mathrm{~cm}$ when no seed was present. Although this difference was statistically significant, $F(1,2)=26.58, p<.04$, the size of the effect was very small and in the direction opposite to that expected if jackdaws could smell the seeds. Therefore we pooled buried-seed and no-seed trials for further analysis. Interlandmark distance had no significant effects on jackdaw's total search accuracy, $F(3,6)=2.77, p>.10$.

We conducted a series of analyses to examine performance when the novel interlandmark distances were introduced during Stage 5 (see Figure 3). A Bird $\times$ Interlandmark Distance analysis showed no significant effects on search error, $F(8,16)<1$. When we divided interlandmark distances into three categories - those used during training, those outside of the training range, and the novel distances within the training range (see Figure 3 ) - the resulting $F$ ratio was again nonsignificant, $F(2,4)<1$.

\section{Discussion}

Although only a few jackdaws completed the experiment, the results obtained are informative. The search task was clearly difficult for them to learn. Although they did show improvement in search accuracy with training, a very large number of training trials were required. During transfer testing, performance on the novel distances did not differ from performance on the training distances. Because so few jackdaws learned the task and search accuracy was very variable and not very good, the results of this experiment must be interpreted with caution. They suggest that jackdaws may possess the ability to learn to search halfway between two landmarks after extended training.

\section{General Discussion}

Nutcrackers, pigeons, and jackdaws all learned to search for buried food near the point halfway between two landmarks when interlandmark distance varied from trial to trial. Nutcrackers and pigeons also transferred accurate search to new interlandmark distances both within and outside the range of distances used during training, but it is difficult to say whether this was also true of the jackdaws. However, although performance was qualitatively similar across species and different from what has previously been reported for pigeons, nutcrackers were more accurate than pigeons or jackdaws. All birds were required to meet an accuracy criterion (mean error less than $25 \mathrm{~cm}$ over 10 days) to move on from the first acquisition stage, but nutcrackers continued to become more accurate after that time, whereas pigeons and jackdaws did not (see Figure 2).

Whenever results from different experiments carried out in different laboratories are compared, especially quantitatively, caution must be exercised. Nonetheless, the mean levels of precision at the end of acquisition achieved by the pigeons and jackdaws lie outside the 95\% confidence interval estimate of the precision of the nutcrackers. This strongly suggests that nutcrackers can perform this task with greater precision than pigeons or jackdaws. This is consistent with the hypothesis that species differences in the precision of spatial search are associated with differences in natural history and not with phylogeny. It is also consistent with the relatively poor radial maze analog performance of jackdaws found by Gould-Beierle (2000), although the performance of nutcrackers was similar to that of scrub jays and pinyon jays in this experiment. The difference between jackdaws and nutcrackers may be a general one of spatial cognition. The relative precision of the nutcracker also suggests that they are particularly suitable animals to use for studies of fine-scaled, landmark-based spatial orientation and navigation.

Despite these quantitative differences, there were substantial qualitative similarities between pigeons and nutcrackers. Previous studies suggested that nutcrackers learned the abstract spatial relationship "middle" (Kamil \& Jones, 1997, 2000), whereas pigeons did not (Spetch et al., 1996, 1997). However, this involved comparisons between experiments in which the two species had been tested with different procedures. In the current experiments, birds of both species were trained and tested with the same protocols, and they showed similar ability to generalize to novel interlandmark distances. This underlines the importance of using tests that are as similar as possible whenever making species comparisons.

No single comparative study of cognition can be definitive. It is always possible that some detail of methodology may have contributed to the pattern of results obtained (Bitterman, 1960). But the differences in spatial accuracy among nutcrackers, pigeons, and jackdaws in the current experiment are consistent with the pattern of results obtained in other comparative studies of pigeons and corvids. Jackdaws have consistently performed better than pigeons on complex, nonspatial tasks (Mackintosh, Wilson, \& Boakes, 1985). Yet they certainly perform no better than pigeons in this spatial task and, in terms of trials to criterion, may even perform worse. Furthermore, nutcrackers have outperformed pigeons in delayed spatial matching (Olson, 1991) and corvids that cache less than nutcrackers in spatial matching (Olson, 1991; Olson et al., 1995) and in a radial maze analog (Kamil et al., 1994). But these differences disappear when these species are tested on nonspatial tasks such as delayed nonmatching (Olson et al., 1995). Species differences associated with dependence on stored food are also found in hippocampal size in corvids (Basil, Kamil, Balda, \& Fite, 1996; Healy \& Krebs, 1992). Similar behavioral (Shettleworth \& Hampton, 1998) and neural (Krebs, Sherry, Healy, Perry, \& Vaccarino, 1989; Sherry, Vaccarino, Buckenham, \& Herz, 1989) differences are found in chickadees, tits, and juncos. This pattern of species differences in behavioral tests and in brain correlations with dependence on scatter-hoarded food indicates that spatial and nonspatial abilities, and associated brain areas, are affected by different selective factors and that these differences are expressed across many different tasks. 


\section{References}

Basil, J. A., Kamil, A. C., Balda, R. P., \& Fite, K. V. (1996). Differences in hippocampal volume among food-storing corvids. Brain, Behavior and Evolution, 47, 156-164.

Biegler, R., McGregor, D., \& Healy, S. D. (1998). How do animals “do" geometry? Animal Behaviour, 57, F4-F8.

Bitterman, M. E. (1960). Toward a comparative psychology of learning. American Psychologist, 15, 704-712.

Cartwright, B. A., \& Collett, T. S. (1983). Landmark learning in bees: Experiments and models. Journal of Comparative Physiology A, 151, 521-543.

Cheng, K. (1986). A purely geometric module in the rat's spatial representation. Cognition, 23, 149-178.

Cheng, K. (1995). Landmark-based spatial memory in the pigeon. In D. L. Medin (Ed.), The psychology of learning and motivation (Vol. 33, (pp. 1-21). San Diego, CA: Academic Press.

Cheng, K., \& Spetch, M. L. (1998). Mechanisms of landmark use in mammals and birds. In S. Healy (Ed.), Spatial representation in animals (p. 188). Oxford, England: Oxford University Press.

Clubb, S. (1997). Aviculture medicine and flock health management. In R. Altman, S. Clubb, G. Dorrestein, \& K. Quesenberry (Eds.), Avian medicine and surgery (pp. 101-116). Philadelphia: W. B. Saunders.

Collett, T., \& Zeil, J. (1996). Flights of learning. Current Directions in Psychological Science, 5, 149-155.

Collett, T., \& Zeil, J. (1998). Places and landmarks: An arthropod perspective. In S. Healy (Ed.), Spatial representations in animals (pp. 1853). Oxford, England: Oxford University Press.

Gould-Beierle, K. (2000). A comparison of four corvid species in a working and reference memory task using a radial maze. Journal of Comparative Psychology, 114, 347-356.

Gould-Beierle, K. L., \& Kamil, A. C. (1996). The use of local and global cues by Clark's nutcrackers, Nucifraga columbiana.. Animal Behaviour, 52, 519-528.

Gould-Beierle, K. L., \& Kamil, A. C. (1998). Use of landmarks in three species of food-storing corvids. Ethology, 104, 361-378.

Healy, S., \& Krebs, J. R. (1992). Food-storing and the hippocampus in corvids: Amount and volume are correlated. Proceedings of the Royal Society of London, B, 248, 241-245.

Hope, S. (1989). Phylogeny of the avian family corvidae. Unpublished doctoral dissertation, City University of New York, New York.

Kamil, A. C., Balda, R. P., \& Olson, D. J. (1994). Performance of four seed-caching corvid species in the radial-arm maze analog. Journal of Comparative Psychology, 108, 385-393.

Kamil, A. C., \& Cheng, K. (2001). Way-finding and landmarks: The multiple-bearings hypothesis. The Journal of Experimental Biology, 204, 103-113.

Kamil, A. C., \& Jones, J. E. (1997). The seed-caching corvid Clark's nutcracker learns geometric relationships among landmarks. Nature, $390,276-279$.

Kamil, A. C., \& Jones, J. E. (2000). Geometric rule learning by Clark's nutcrackers (Nucifraga columbiana). Journal of Experimental Psychology: Animal Behavior Processes, 26, 439-453.

Kelly, D. M., Spetch, M. L., \& Heth, C. D. (1998). Pigeons' (Columba livia) encoding of geometric and featural properties of a spatial environment. Journal of Comparative Psychology, 112, 259-269.
Krebs, J. R., Sherry, D. F., Healy, S. D., Perry, V. H., \& Vaccarino, A. L. (1989). Hippocampal specialization of food-storing birds. Proceedings of the National Academy of Sciences, USA, 86, 1388-1392.

Mackintosh, N. J. (1988). Approaches to the study of animal intelligence. British Journal of Psychology, 79, 509-525.

Mackintosh, N. J., Wilson, B., \& Boakes, R. A. (1985). Differences in mechanisms of intelligence among vertebrates. In L. Weiskrantz (Ed.), Animal intelligence (pp. 53-63). Oxford, England: Clarendon Press.

Margules, J., \& Gallistel, C. R. (1988). Heading in the rat: Determination by environmental shape. Animal Learning and Behavior, 16, 404-410.

O'Keefe, J., \& Burgess, N. (1996). Geometric determinants of the place fields of hippocampal neurons. Nature, 381, 425-428.

Olson, D. J. (1991). Species differences in spatial memory among Clark's nutcrackers, scrub jays, and pigeons. Journal of Experimental Psychology: Animal Behavior Processes, 17, 363-376.

Olson, D. J., Kamil, A. C., Balda, R. P., \& Nims, P. J. (1995). Performance of four seed-caching corvid species in operant tests of nonspatial and spatial memory. Journal of Comparative Psychology, 109, 173-181.

Sherry, D. F., Vaccarino, A. L., Buckenham, K., \& Herz, R. (1989). Hippocampal size in food-storing birds. Brain, Behavior and Evolution, 34, 308-317.

Shettleworth, S. J. (1998). Cognition, evolution and behavior. Oxford, England: Oxford University Press.

Shettleworth, S. J., \& Hampton, R. H. (1998). Adaptive specializations of spatial cognition in food storing birds? Approaches to testing a comparative hypothesis. In R. P. Balda, I. M. Pepperberg, \& A. C. Kamil (Eds.), Animal cognition in nature (pp. 65-98). San Diego, CA: Academic Press.

Spetch, M. L., Cheng, K., \& MacDonald, S. E. (1996). Learning the configuration of a landmark array: I. Touch screen studies with pigeons and humans. Journal of Comparative Psychology, 110, 55-68.

Spetch, M. L., Cheng, K., MacDonald, S. E., Linkenhoker, B., Kelly, D., \& Doerkson, S. (1997). Use of landmark configuration by pigeons and humans: II. Generality across search tasks. Journal of Comparative Psychology, 111, 14-24.

Tommasi, L., \& Vallortigara, G. (2000). Searching for the center: Spatial cognition in the domestic chick. Journal of Experimental Psychology: Animal Behavior Processes, 26, 477-486.

Vallortigara, G., Zanforlin, M., \& Pasti, G. (1990). Geometric modules in animals' spatial representations: A test with chicks (Gallus gallus domesticus). Journal of Comparative Psychology, 104, 248-254.

Vander Wall, S. B., \& Balda, R. P. (1977). Coadaptations of the Clark's nutcracker and the pinon pine for efficient seed harvest and dispersal. Ecological Monographs, 47, 89-111.

Wilson, B., Mackintosh, N. J., \& Boakes, R. A. (1985). Transfer of relational rules in matching and oddity learning by pigeons and corvids. Quarterly Journal of Experimental Psychology: Comparative and Physiological Psychology, 37B, 313-332.

Wright, A. A., Cook, R. G., Rivera, J. J., Sands, S. F., \& Delius, J. D. (1988). Concept learning by pigeons: Matching-to-sample with trial-unique video picture stimuli. Animal Learning and Behavior, $16,436-444$. 